\title{
TGF- $\beta$ switches from tumor suppressor to prometastatic factor in a model of breast cancer progression
}

\author{
Binwu Tang, ${ }^{1}$ Mary Vu, ${ }^{1}$ Timberly Booker, ${ }^{1}$ Steven J. Santner, ${ }^{2}$ Fred R. Miller, ${ }^{2}$ \\ Miriam R. Anver, ${ }^{3}$ and Lalage M. Wakefield ${ }^{1}$ \\ ${ }^{1}$ Laboratory of Cell Regulation and Carcinogenesis, National Cancer Institute, Bethesda Maryland, USA \\ ${ }^{2}$ Barbara Ann Karmanos Cancer Institute, School of Medicine, Wayne State University, Detroit, Michigan, USA \\ ${ }^{3}$ Pathology/Histotechnology Laboratory, Scientific Applications International Corp., National Cancer Institute at Frederick, \\ Frederick, Maryland, USA
}

\begin{abstract}
The TGF- $\beta$ signaling network plays a complex role in carcinogenesis because it has the potential to act as either a tumor suppressor or a pro-oncogenic pathway. Currently, it is not known whether TGF- $\beta$ can switch from tumor suppressor to pro-oncogenic factor during the course of carcinogenic progression in a single cell lineage with a defined initiating oncogenic event or whether the specific nature of the response is determined by cell type and molecular etiology. To address this question, we have introduced a dominant negative type II TGF- $\beta$ receptor into a series of genetically related human breast-derived cell lines representing different stages in the progression process. We show that decreased TGF- $\beta$ responsiveness alone cannot initiate tumorigenesis but that it can cooperate with an initiating oncogenic lesion to make a premalignant breast cell tumorigenic and a low-grade tumorigenic cell line histologically and proliferatively more aggressive. In a high-grade tumorigenic cell line, however, reduced TGF- $\beta$ responsiveness has no effect on primary tumorigenesis but significantly decreases metastasis. Our results demonstrate a causal role for loss of TGF- $\beta$ responsiveness in promoting breast cancer progression up to the stage of advanced, histologically aggressive, but nonmetastatic disease and suggest that at that point TGF- $\beta$ switches from tumor suppressor to prometastatic factor.
\end{abstract}

J. Clin. Invest. 112:1116-1124 (2003). doi:10.1172/JCI200318899.

\begin{abstract}
Introduction
TGF- $\beta$ s and their cognate receptors are expressed in essentially all tissues and are thought to play complex roles in carcinogenesis (reviewed in refs. 1-4). This complexity probably reflects the broad range of biological activities that are modulated by TGF- $\beta$ s and the many different cellular players in the carcinogenic process. Currently, the prevailing hypothesis is that TGF- $\beta$ s function as tumor suppressors early in epithelial carcinogenesis but that they switch to a pro-oncogenic role later in progression $(2,3)$. Piecemeal evidence in support of this hypothesis has been gathered from many different experimental systems. In no case, however, has it been demonstrated that the endogenous TGF- $\beta$ signaling network can indeed switch roles during the course of carcinogenic progression in a given cell lineage with a defined initiating oncogenic event. The alternative possibility would be that TGF- $\beta$ plays differing roles depending on the
\end{abstract}

Received for publication May 12, 2003, and accepted in revised form July 29, 2003.

Address correspondence to: Lalage Wakefield, Laboratory of Cell Regulation and Carcinogenesis, National Cancer Institute, Building 41, Room C629, 41 Library Drive, MSC 5055, Bethesda, Maryland 20892-5055, USA. Phone: (301) 496-8351; Fax: (301) 496-8395; E-mail: wakefiel@dce41.nci.nih.gov.

Conflict of interest: The authors have declared that no conflict of interest exists.

Nonstandard abbreviations used: type II TGF- $\beta$ receptor (T $\beta$ RII); ductal carcinoma in situ (DCIS); dominant negative type II TGF- $\beta$ receptor (DNR); control (CON). specific molecular etiology of the tumor or the specific cell type of origin. These two possibilities have different implications for the design of novel preventive or therapeutic strategies that target the TGF- $\beta$ system.

The proposed dual role for TGF- $\beta$ in carcinogenesis has its basis in both experimental and clinical observations. Work in vitro has shown that TGF- $\beta$ s are highly potent inhibitors of proliferation for nearly all epithelia. In addition, TGF- $\beta$ s can induce apoptosis, promote replicative senescence, maintain genomic stability, induce a negative angiogenic regulator profile, and interfere with cellular immortalization. All these properties could contribute to tumor suppression (reviewed in refs. 2, 3). Consistent with a tumor-suppressor role for TGF- $\beta$, analysis of clinical samples and tumor-derived cell lines suggests that reduction or alteration in epithelial responsiveness to TGF- $\beta$ is a common occurrence during carcinogenic progression in many tissues (5). On the other hand, overexpression of TGF- $\beta$ ligands is a feature of many advanced human tumors, and elevated TGF- $\beta$ expression almost invariably associates with indicators of poor prognosis, consistent with a pro-oncogenic role for TGF- $\beta$ in latestage cancer (6). Pro-oncogenic effects that would promote tumor progression include direct effects of TGF- $\beta$ s on the tumor cells themselves, such as the enhancement of invasion and motility, as well as indirect effects that generate a more permissive tumor stroma, including effects on angiogenesis and immune surveillance (reviewed in refs. 2,3). 
Clinical studies of breast cancer have shown that perturbations in the normal functioning of the TGF- $\beta$ system occur at several distinct stages during disease progression. Reduced or heterogeneous expression of the type II TGF- $\beta$ receptor (T $\beta$ RII) is seen in a subset of patients with breast epithelial hyperplasia without atypia and is associated with an increased risk of subsequently developing invasive breast cancer (7). Thus, diminished TGF- $\beta$ responsiveness is a marker for risk of malignant conversion in an otherwise benign proliferative lesion. Furthermore, reduced T $\beta$ RII expression in ductal carcinoma in situ (DCIS) and invasive breast cancer correlates with higher mitotic count, higher tumor grade, and clinical stage, consistent with a tumor-suppressor role for TGF- $\beta$ at this later stage, too (8). As for carcinomas in other organs, however, TGF- $\beta$ levels are increased locally and systemically in advanced breast cancers, particularly at the leading invasive edge of the tumor and in metastases $(9-12)$. The elevated expression of TGF- $\beta$ associates with disease progression and correlates with node positivity and metastasis, suggesting that at this late stage, TGF- $\beta$ is now promoting carcinogenic progression $(10,11)$.

In the present study we had two major goals. The first was to establish a causal connection between the reduced TGF- $\beta$ responsiveness and disease progression that is seen in clinical breast samples. The second was to test the important, and more general, hypothesis that TGF- $\beta$ can switch from tumor suppressor to prometastatic factor during the course of carcinogenic progression in a given cell lineage. To do this, we have used a unique series of human breast epithelial cell lines representing four different stages of the carcinogenic process. All the cell lines are derivatives of the immortal, but otherwise normal, MCF10A cell line, which was originally derived from benign breast tissue from a woman with fibrocystic disease (13). They span the spectrum of progression from relatively normal breast epithelial cells through to highly malignant metastatic cancer cells $(14,15)$. To model the reduced expression of T $\beta R I I$ that is seen clinically, we introduced a dominant negative TGFßRII receptor into all four cell lines, and tested for effects on tumorigenicity in vivo. Our data strongly support the hypothesis that TGF- $\beta$ switches from tumor suppressor to prometastatic factor during breast cancer progression.

\section{Methods}

Cell lines. All the cell lines are derivatives of the immortal but otherwise normal MCF10A breast epithelial cell line (13). Transfection of MCF10A with activated HRAS and selection by xenografting gave the MCF10AT1k.cl2 cell line, which gives rise to premalignant lesions with the potential for neoplastic progression (14). MCF10Ca1h and MCF10CA1a.cl1 were derived from occasional carcinomas arising from xenografts of MCF10AT1k.cl2. MCF10Ca1h gives predominantly well-differentiated carcinomas on xenografting, while MCF10Ca1a gives rise to relatively undifferentiated carcinomas and metastasizes to the lung upon injection into the tail vein (15). For the sake of simplicity, in the present study we refer to these cell lines as M-I through M-IV, and the properties and derivation of these cells are summarized in Table 1. The cell lines were obtained from Fred Miller and Steven Santner at the Barbara Ann Karmanos Cancer Institute. The M-III and M-IV cells were maintained in DMEM/F-12 supplemented with $5 \%$ horse serum and $1 \%$ penicillin/streptomycin at $37^{\circ} \mathrm{C}, 5 \% \mathrm{CO}_{2}$. The culture medium for the M-I and M-II was similar but additionally included $10 \mu \mathrm{g} / \mathrm{ml}$ insulin, $20 \mathrm{ng} / \mathrm{ml}$ EGF, $0.5 \mu \mathrm{g} / \mathrm{ml}$ hydrocortisone, and $100 \mathrm{ng} / \mathrm{ml}$ cholera toxin.

Retroviral transduction. The human T $\beta$ RII sequence, between nucleotides 1 and 656, was PCR amplified and ligated into the EcoRI/BamHI sites of the pcDNA3.1myc-HisB(+) vector (Invitrogen Corp., Carlsbad, California, USA). This dominant negative form of the receptor (DNR) with Myc-HisB tag was then PCR amplified and subcloned into the proretroviral vector pLPCX (BD Biosciences Clontech, Palo Alto, California, USA) to generate the proretroviral construct pLPCDNR. Replication-defective infectious retrovirus was generated by transfecting proretroviral plasmids into A-BOSC cells (16). Exponentially growing M-I through M-IV cells were transduced with pLPCX control retrovirus or PLPC-DNR and were maintained under puromycin selection $(2 \mu \mathrm{g} / \mathrm{ml})$ for 5 days. In vivo experiments were performed within $2-3$ weeks of transduction using pools of transduced cells.

Ligand-affinity cross-linking, growth-inhibition assays, and Western blot analysis. For detection of cell surface TGF- $\beta$ receptors, cell monolayers at $70-80 \%$ confluence were

\section{Table 1}

Summary of properties of MCF10A-derived cell lines

\begin{tabular}{|c|c|c|c|c|}
\hline Cell line designation & MCF10A1 (M-I) & MCF10AT1k.cl2 (M-II) & MCF10CA1h (M-III) & MCF10CA1a.cl1 (M-IV) \\
\hline $\begin{array}{l}\text { Stage of breast } \\
\text { carcinogenesis modeled }\end{array}$ & Normal epithelium & $\begin{array}{l}\text { Oncogenically initiated } \\
\text { premalignant epithelium }\end{array}$ & Low-grade carcinoma & $\begin{array}{l}\text { High-grade metastatic } \\
\text { carcinoma }\end{array}$ \\
\hline Description of line & $\begin{array}{l}\text { Spontaneously immortalized } \\
\text { line from nonmalignant } \\
\text { human breast epithelium }\end{array}$ & $\begin{array}{l}\text { Derived from MCF10A cells } \\
\text { transfected with activated } \\
\text { HRAS oncogene (MCF10AT) } \\
\text { and passaged through mice as } \\
\text { persistent lesions with features } \\
\text { of proliferative breast disease }\end{array}$ & $\begin{array}{l}\text { Line derived from a } \\
\text { xenograft implant of } \\
\text { MCF10AT cells that } \\
\text { progressed to } \\
\text { carcinoma }\end{array}$ & $\begin{array}{l}\text { Line derived from } \\
\text { xenograft implants of } \\
\text { MCF10AT cells that } \\
\text { progressed to carcinoma }\end{array}$ \\
\hline $\begin{array}{l}\text { Tumorigenicity on } \\
\text { xenografting into } \\
\text { nude mice }\end{array}$ & Nontumorigenic & $\begin{array}{l}\text { Initially form simple ducts } \\
\text { that can progress to benign } \\
\text { hyperplastic lesions and } \\
\text { occasionally carcinomas }\end{array}$ & $\begin{array}{l}\text { Form predominantly } \\
\text { well-differentiated } \\
\text { carcinomas }\end{array}$ & $\begin{array}{l}\text { Form poorly differentiated } \\
\text { carcinomas; metastatic to } \\
\text { lung in tail vein-injection } \\
\text { assay }\end{array}$ \\
\hline
\end{tabular}


affinity cross-linked with $100 \mathrm{pM}{ }^{125}$ I-labeled TGF- $\beta 1$ (Perkin-Elmer Life Sciences, Boston, Massachusetts, USA) as described previously (17). To confirm identity of the DNR, some labeled samples were immunoprecipitated with anti-Myc Ab (Invitrogen Corp.) prior to loading. Cell proliferation was determined by ${ }^{3} \mathrm{H}$-thymidine incorporation essentially as described (17). For Western blots, cell monolayers at $30-40 \%$ confluence were switched from normal growth medium to DMEM/F-12 $1 \%$ calf serum for 18 hours, then cells were treated with $5 \mathrm{ng} / \mathrm{ml} \mathrm{TGF-} \beta$ and harvested at various time points after treatment. Ab's were from the following sources: fibronectin and actin, Santa Cruz Biotechnology Inc. (Santa Cruz, California, USA); c-Myc, Active Motif LLC (Carlsbad, California, USA); Smad2 and Smad3, Zymed Laboratories Inc. (South San Francisco, California, USA); phospho-Smad2, Upstate Biotechnology Inc. (Lake Placid, New York, USA); phospho-Smad3, a generous gift of Michael Reiss (Cancer Institute of New Jersey, Nutley, New Jersey, USA).

Tumorigenesis. M-III and M-IV cells were suspended in DMEM/F-12 at a density of $5 \times 10^{6}$ cells $/ \mathrm{ml}$ and $10^{6}$ cells/ml, respectively, while M-I and M-II cells were suspended in a 1:1 vol/vol mix of DMEM/F-12 Matrigel (BD BioSciences, Bedford, Massachusetts, USA) at a density of $5 \times 10^{7}$ cells $/ \mathrm{ml}$. Five-week-old female athymic
$\mathrm{NCr} n u / n u$ mice were inoculated subcutaneously on the hind flank with $0.2 \mathrm{ml}$ of the cell suspension. Palpable lesions were measured twice weekly with calipers, and tumor volumes were calculated by the formula $(S \times S \times L)$ $\times 0.52$, where $S$ and $L$ are the short and long dimensions, respectively. Mice were cared for humanely according to the U.S. Public Health Policy on the Care and Use of Animals and the Guide for the Care and Use of Laboratory Animals. Mice were euthanized with $\mathrm{CO}_{2}$ when tumors reached $2 \mathrm{~cm}$ in diameter, or if tumors ulcerated, or the mice showed significant morbidity. Half of each tumor was snap-frozen for molecular analysis and half was fixed in neutral-buffered formalin for histology. All tumors were examined by a board-certified veterinary pathologist (M.R. Anver). Xenografts were graded on a scale of 0 (minimum) to 4 (marked) for the presence of mitoses and for the extent of atypia. Atypia consisted of sheets and nests of poorly differentiated epithelial cells with indistinct cytoplasmic borders and large pleomorphic vesicular nuclei.

Metastasis. To determine metastatic potential, 5-weekold female nude mice were injected intravenously with $10^{6}$ cells in $0.2 \mathrm{ml}$ of DMEM/F-12 in the tail vein. Quantitation of metastases was performed on representative cross-sections of formalin-fixed, paraffinembedded lungs, stained with hematoxylin and eosin.
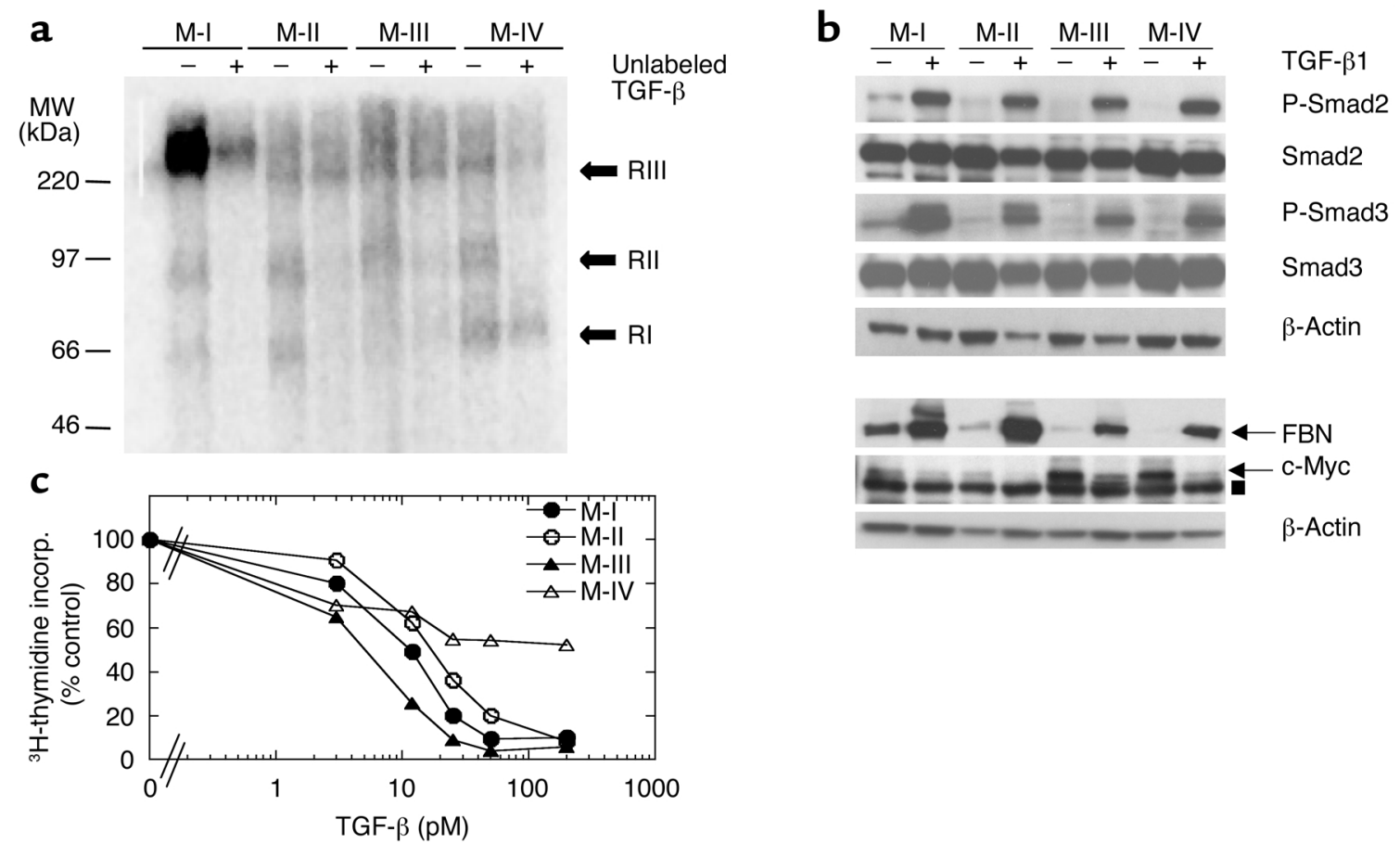

\footnotetext{
Figure 1

TGF- $\beta$ receptor expression and responsiveness in the four human breast epithelial cell lines. (a) Ligand affinity cross-linking. Cell lines were affinity labeled with 100 pM ${ }^{125}$ I-labeled TGF- $\beta 1$ in the absence $(-)$ or presence $\left(^{+}\right)$of a 50 -fold molar excess of unlabeled TGF- $\beta 1$. The migration positions of the endogenous TGF- $\beta$ receptors (RI, RII, and RIII) are indicated. MW, molecular weight. (b) Smad phosphorylation and regulation of gene expression. Cells were treated with $2 \mathrm{ng} / \mathrm{ml}$ TGF- $\beta$ and assessed by Western blot analysis for extent of Smad phosphorylation (at $t=15$ minutes), and effects on fibronectin (FBN) and c-Myc expression (at $t=24$ hours). For the c-Myc blot, the arrow indicates the c-Myc band and the filled square indicates a nonspecific band. (c) Growth-inhibitory effects of TGF- $\beta 1$. Cell proliferation in the presence of increasing amounts of TGF- $\beta 1$ was quantitated by ${ }^{3} \mathrm{H}$-thymidine incorporation (incorp.). Results are the means for three determinations normalized to the controls with no added TGF- $\beta$ for each cell type.
} 
Immunohistochemistry and quantitation of proliferation and apoptosis in tumors. All mice were injected with 150 $\mathrm{mg} / \mathrm{kg}$ BrdU 1 hour before euthanasia for determination of proliferative indices. Formalin-fixed sections were immunostained using an anti-BrdU mAb (DAKO Corp., Carpinteria, California, USA). The number of BrdU-labeled cells was determined for 20-40 randomly chosen high-power fields for each tumor. Similarly, the number of apoptotic cells was quantitated following TUNEL staining using the ApoTag kit (Oncor Inc., Gaithersburg, Maryland, USA). Immunohistochemistry was performed on a sample of M-III tumors for pan-actin and S100 (DAKO Corp.) and cytokeratin 14 (The Binding Site Inc., San Diego, California, USA).

\section{Results}

The cell lines all express TGF- $\beta$ receptors and respond to TGF- $\beta$ in vitro. We began by characterizing TGF- $\beta$ receptor expression and TGF- $\beta$ responsiveness in the breast cell lines whose properties are summarized in Table 1. As shown in Figure 1a, all four cell lines express similar levels of the signaling receptors, T $\beta R I$ and T $\beta R I I$, as determined by ligand-affinity cross-linking. M-I sometimes showed much higher levels of the accessory binding protein T $\beta$ RIII than did the other cell lines, but this was not a consistent finding. All four cell lines expressed similar levels of the downstream signaling molecules, Smad2 and Smad3, and TGF- $\beta$ induced phosphorylation of both Smads to a similar extent in all four lines (Figure 1b). Similarly, TGF- $\beta$ could induce expression of fibronectin and suppress expression of c-Myc in all four lines, though basal expression of c-Myc varied somewhat between the lines (Figure 1b). Finally, all four cell lines were growth inhibited by TGF- $\beta 1$ in a dose-dependent manner, with an $\mathrm{ED}_{50}$ of approximately 10-20 pM (Figure 1c). Whereas the proliferation of M-I, M-II, and M-III could all be completely inhibited by TGF- $\beta$, however, the most aggressive cell line, M-IV, was only partially inhibited $(\sim 40 \%)$ at the highest TGF- $\beta$ concentrations. Thus, all four cell lines expressed functional TGF- $\beta$ receptors, phosphorylated the Smad signal transduction components similarly, showed normal regulation of hallmark TGF- $\beta$-regulated genes, and demonstrated at least some sensitivity to growth inhibition by TGF- $\beta$.

The DNR blocks TGF- $\beta$ function in vitro. All four cell lines were transduced with a control retrovirus $(\mathrm{CON})$ or a retrovirus expressing the DNR and were characterized for TGF- $\beta$ responses in vitro. Representative results with the M-III cells are shown in Figure 2. Western blot analysis of whole cell lysates, using an anti-Myc Ab to detect the tagged DNR, showed a band at $40 \mathrm{kDa}$, indicating that the core $29 \mathrm{kDa}$ DNR protein was expressed and glycosylated in the transduced cells (Figure 2a). Ligand-affinity cross-linking using ${ }^{125}$ I-labeled TGF- $\beta 1$ followed by immunoprecipitation with anti-Myc Ab confirmed that the DNR trafficked correctly to the cell surface and was capable of binding TGF- $\beta$ (Figure $2 \mathrm{~b}$ ).

We then tested whether the DNR construct could block phosphorylation of downstream signaling com- ponents of the Smad family (2). Interestingly, expression of the DNR had only a partial effect on the phosphorylation of Smad2 and Smad3 at early time points, but it caused a more complete blocking by 18 hours after addition of TGF- $\beta$ (Figure $2 \mathrm{c}$ ). Despite this apparently incomplete blocking of TGF- $\beta$ signal transduction, the DNR typically decreased the induction of fibronectin and the repression of c-Myc protein by TGF- $\beta$ to approximately $15 \%$ of that seen in control cells (Figure $2 \mathrm{~d}$ ). M-III DNR cells were also largely resistant to
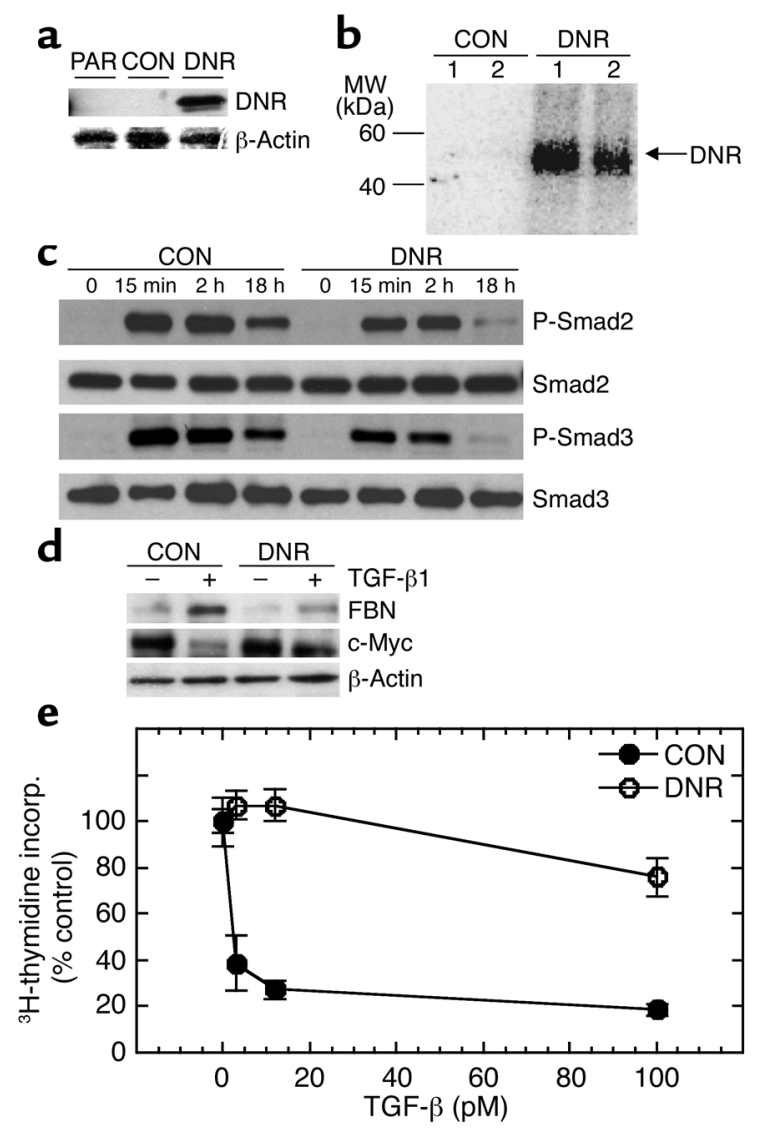

Figure 2

Blockade of TGF- $\beta$ responses in vitro by transduction with the DNR. (a) Expression of the DNR in transduced cells. DNR expression in transduced $\mathrm{M}$-III cells was determined by Western blot analysis probing for the Myc tag on the DNR. $\beta$-Actin protein was used for normalization. (b) Ability of the DNR to bind TGF- $\beta$. M-III cells were affinity labeled with ${ }^{125}$ I-TGF- $\beta 1$. Following cross-linking, lysates were immunoprecipitated with anti-Myc Ab for visualization of ligand bound to the DNR. (c) Effect of DNR on Smad phosphorylation by TGF- $\beta$. M-III cells were treated with $5 \mathrm{ng} / \mathrm{ml} \mathrm{TGF-} \beta 1$ for various times, and Smad protein expression and phosphorylation were analyzed by Western blot. P-Smad, phosphos-Smad. (d) Effect of DNR on gene-regulation responses to TGF- $\beta 1$. M-III cells were treated with $5 \mathrm{ng} / \mathrm{ml}$ TGF- $\beta 1$ or vehicle alone for 18 hours, and fibronectin (FBN) and c-Myc expression were analyzed by Western blot. (e) Effect of DNR on growth inhibition induced by TGF- $\beta 1$. Growth inhibition in response to TGF- $\beta 1$ was measured by $\left[{ }^{3} \mathrm{H}\right]$-thymidine incorporation. All results are the mean \pm SD for three determinations and are normalized to no TGF- $\beta$ controls for each sample. PAR, untransduced parental M-III cells; CON, M-III cells transduced with PLPCX control retrovirus; DNR, M-III cells transduced with PLPC-DNR. 


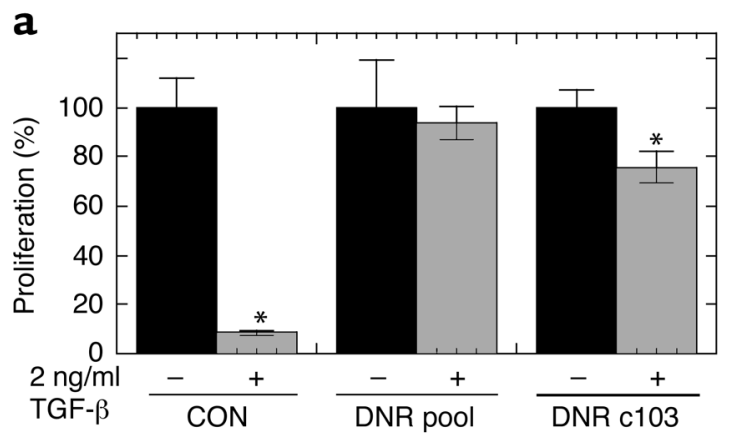

b
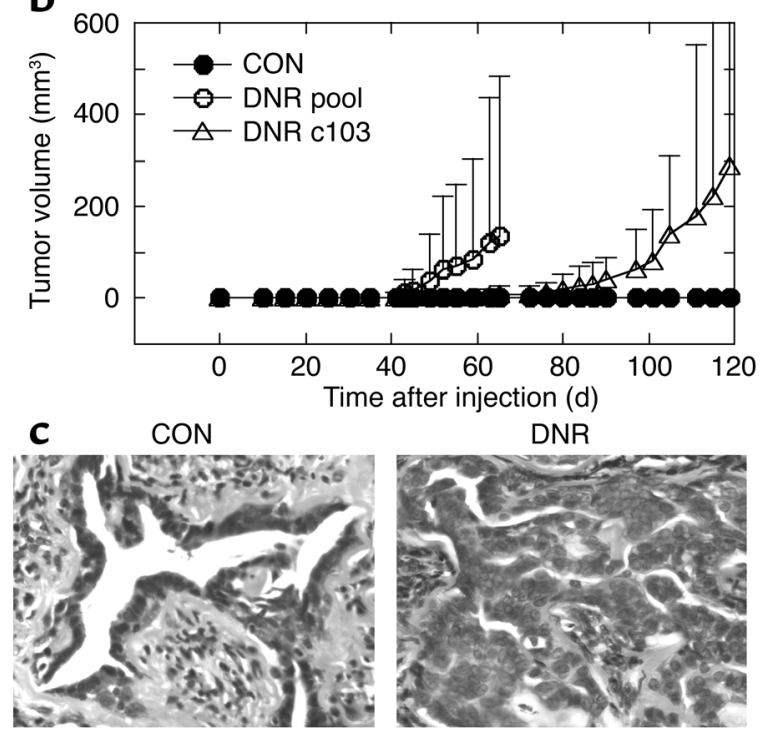

growth inhibition, with treatment up to $100 \mathrm{pM}$ of TGF- $\beta 1$ (Figure 2e). In some experiments, complete loss of growth-inhibitory response was observed in the pools of DNR-transduced cells, whereas in others, or in cloned transductants, some residual response was seen at high TGF- $\beta$ concentrations (Figure $2 \mathrm{e}$ and Figure $3 \mathrm{a}$ ). Thus, in general, the DNR reduced but did not totally block the various biological responses to TGF- $\beta$.

Loss of TGF- $\beta$ response causes malignant transformation of oncogenically initiated premalignant breast epithelial cells. To determine the effect of reduced TGF- $\beta$ responsiveness on the tumorigenicity of the different cell lines in vivo, we xenografted all four cells and their DNR-expressing derivatives into athymic nude mice. For the M-I immortalized "normal" breast epithelial cell line, no lesions formed up to 1 year after injection, whether or not they expressed the DNR (total of six injection sites for each of the DNR and CON genotype groups; data not shown). This result suggests that loss of TGF- $\beta$ response is not an initiating event for breast carcinogenesis.

For the M-II cell line, which is an oncogenic HRASinitiated premalignant line, tumorigenicity was determined both for a pool of transduced cells in which TGF- $\beta$ response was fully blocked (DNR pool) and a clone that showed some residual TGF- $\beta$ responsiveness (DNR c103) (Figure 3a). No palpable lesions formed for the duration of the experiment in parental cells (not

\section{Figure 3}

Decreased TGF- $\beta$ responsiveness increases the probability of malignant conversion for the premalignant breast cell line M-II. (a) TGF- $\beta$ responsiveness of $\mathrm{M}-\mathrm{II}$ transductants in vitro. The proliferation of $\mathrm{M}-\mathrm{II}$ transductants in the presence $(+)$ and absence $(-)$ of $2 \mathrm{ng} / \mathrm{ml}$ TGF- $\beta 1$ was determined by incorporation of ${ }^{3} \mathrm{H}$-thymidine. Results are the mean \pm SD of three determinations and are normalized to the no TGF- $\beta$ control in each case. ${ }^{*} P<0.01$. (b) Tumor growth kinetics. Five-week-old female athymic nude mice were inoculated subcutaneously on each hind flank with retrovirally transduced M-II cells $\left(5 \times 10^{6}\right.$ cells/site; ten sites per genotype). (c) Histology of lesions formed by M-II transductants. M-II CON cells formed cystic ductal structures, while MII-DNR formed glandular carcinomas. CON, M-II cells transduced with pLPCX; DNR-pool, pooled M-II cells transduced with PLPC-DNR at levels that fully blocked TGF- $\beta$ growth-inhibitory responses; DNR-c103, a clone of M-II cells transduced with pLPC-DNR that retained partial TGF- $\beta$ responsiveness.

shown), nor in cells transduced with control retrovirus (Figure $3 \mathrm{~b}$ ). At $17 \%$ of the injection sites ( 3 of 18 ), cystic ductal structures or hyperplasias were seen after 120 days, but no tumors were observed (Figure 3c, CON). In contrast, the M-II DNR cells formed rapidly growing lesions with a latency that depended on the level of effectiveness of the DNR. For the pool of DNR-transduced cells with no growth-inhibitory response to TGF- $\beta$, palpable lesions were detected with a latency of approximately 40 days (Figure 3a). By 65 days, measurable lesions were present at six of ten injection sites. Histologically, the lesions formed were all either glandular or papillary carcinomas (Figure $3 c$, DNR). The difference in tumor incidence between the CON and DNR groups was statistically significant $(P<0.001$; Fisher exact test). Similar results were obtained in a second independent experiment (data not shown). For the clone of DNR-transduced cells in which the growthinhibitory response was only partially blocked (DNR clone 103), tumors arose with a longer latency (63 days) but a similar incidence (seven of ten) (Figure 3b). In summary, these data indicate that decreased TGF- $\beta$ responsiveness can cooperate with an initiating oncogenic lesion to increase the frequency of malignant transformation of a breast epithelial cell. This observation is consistent with a tumor-suppressor role for TGF- $\beta$ in the premalignant breast epithelium.

Loss of TGF- $\beta$ response increases the aggressiveness of a lowgrade breast carcinoma. The M-III cell line formed slowgrowing tumors upon xenografting into nude mice (Figure 4a). In comparison, tumors formed by the M-III DNR cells had a shorter latency (approximately 14 days compared with approximately 25 days) and grew faster (Figure 4a). Mean tumor weight at 30 days after injection was $1.9 \pm 1.2 \mathrm{~g}$ for M-III DNR tumors and $0.48 \pm 0.56 \mathrm{~g}$ for M-III CON tumors $(P=0.003$, twotailed $t$ test for independent samples). This result was confirmed by two additional independent experiments (data not shown).

All of the lesions formed by both genotype groups were carcinomas. All tumors had admixtures of three distinct morphologic types (Figure 4b). The more well- 
a

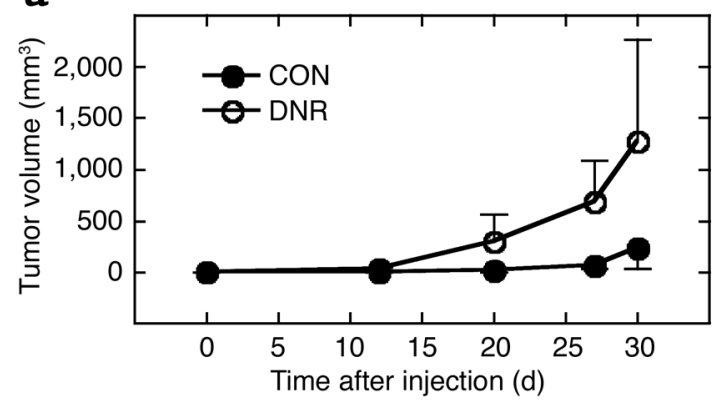

b

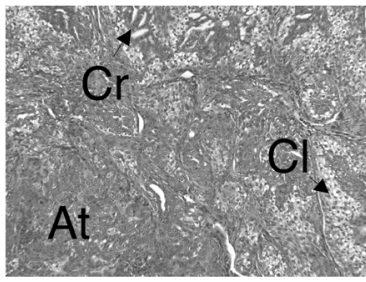

d

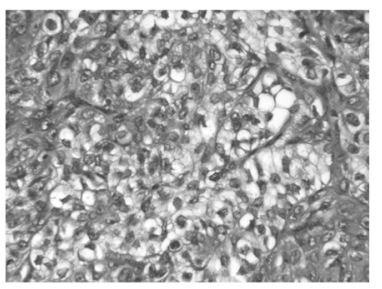

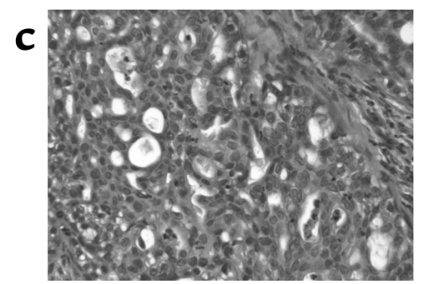

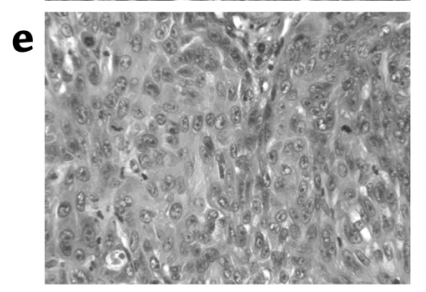

f

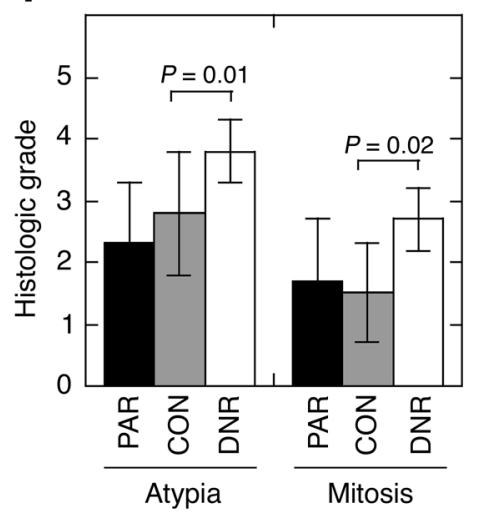

g

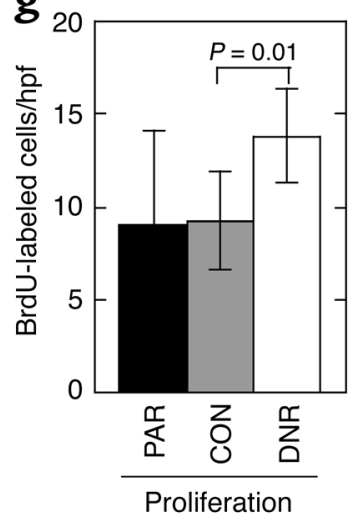

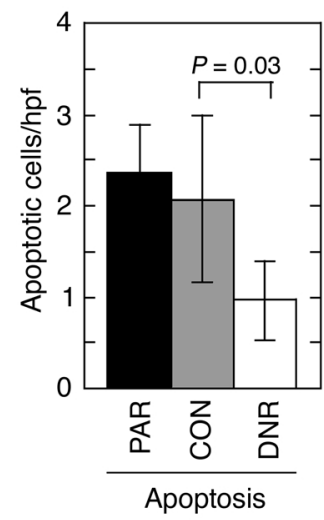

Figure 4

Decreased TGF- $\beta$ responsiveness increases the tumor growth rate and histological grade of the low-grade breast carcinoma line M-III. (a) Tumor growth kinetics. Nude mice were inoculated subcutaneously on each hind flank with retrovirally transduced M-III cells ( $10^{6}$ cells/site; 10 sites/genotype). Untransduced parental M-III cells ( $n=4$, not shown) gave results essentially identical to M-III CON. (b-e). Histology of lesions formed by M-III transductants. Tumors of both genotypes were admixtures of three morphologic types. (b) M-III DNR tumor showing all three morphologies ( $\mathrm{Cr}$, cribriform structures; $\mathrm{Cl}$, clear cells; At, area of atypia); (c) cribriform glands in an M-III CON tumor; (d) clear cell area in an M-III CON tumor; (e) area of atypia from an M-III DNR tumor. Magnification: $\times 100$ (b) and $\times 400(\mathbf{c}-\mathbf{e})$. (f) Atypia and mitosis grades in M-III tumors. Histological sections were graded from 0 to 4 independently for extent of atypia and for frequency of mitoses as detailed in Methods. (g) Proliferation and apoptosis rates in M-III tumors. Tumor cell proliferation was quantitated by counting BrdU-labeled nuclei on histologic sections, and apoptotic cells were quantitated by TUNEL assay. Results are the mean \pm SD for a minimum of five tumors of each genotype. PAR, parental untransduced cells; CON, cells transduced with pLPCX; DNR, cells transduced with pLPC-DNR. hpf, high-power field.

differentiated areas were composed of irregular cribriform glands lined by low cuboidal epithelium (Figure 4c). Clear cell areas consisted of closely apposed round cells with clear cytoplasm and hyperchromatic nuclei (Figure 4d). By immunohistochemistry, clear cells were variably positive for S100 and cytokeratin 14 and negative for actin, suggesting that they are of myoepithelial origin. Poorly differentiated areas were composed of nests and sheets of epithelial cells with large, pleomorphic vesicular nuclei with multiple nucleoli, and mitotic figures were most frequent in these areas (Figure 4e). Using a grading system for the degree of atypia based on the ratio of areas of poorly differentiated cells to areas of cribriform glands, the atypia grade was significantly increased in the M-III DNR compared with M-III CON tumors, as was the mitosis grade (Figure 4f). There was also an increase in the proliferation index as determined by BrdU labeling (Figure $4 \mathrm{~g}$ ) and a significant decrease in apoptosis in the M-III DNR tumors (Figure 4g). Overall, the results suggest that diminished TGF- $\beta$ responsiveness causes a well-differentiated breast carcinoma to grow faster and become less differentiated, suggesting that TGF- $\beta$ plays a tumor-suppressor role in low-grade breast cancer.

Loss of TGF- $\beta$ response does not affect primary tumorigenesis, but suppresses metastasis in a high-grade breast carcinoma. The most aggressive cell line, M-IV, formed rapidly growing, poorly differentiated tumors on xenografting into nude mice. Introduction of the DNR into these cells did not change the growth rate (Figure 5a) or histology of the primary tumors (not shown). All M-IV tumors were a mixture of poorly differentiated sheets of pleiomorphic cells and areas of "clear cells," but without the cribriform glands that were seen in the M-III tumors. To determine whether loss of TGF- $\beta$ response would affect metastatic efficiency, genetically 


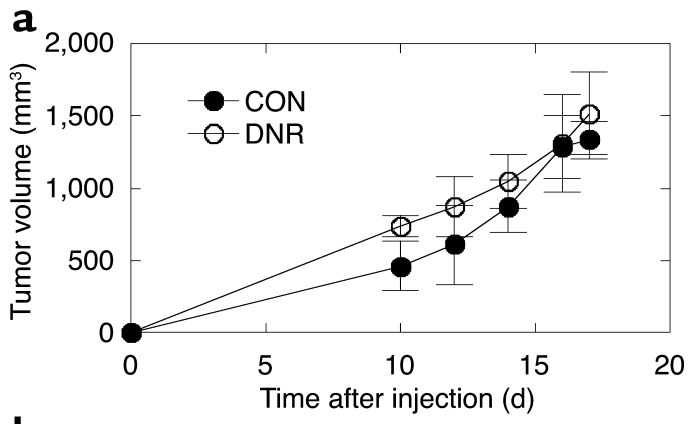

b

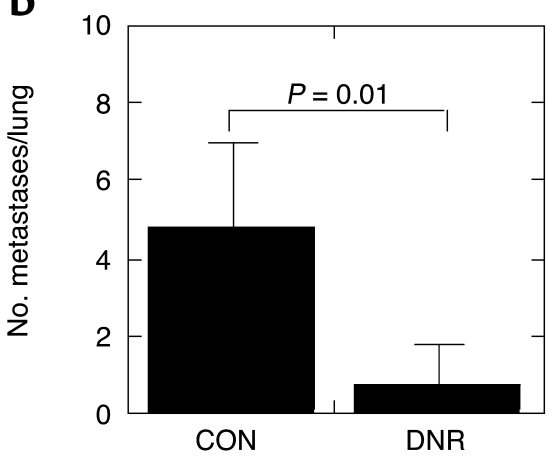

modified M-IV cells were injected into the tail vein of nude mice. While all mice in the control group (five of five) developed lung metastases by 8 weeks after injection, only $40 \%$ of the mice injected with the M-IV DNR cells (four of ten) developed metastases in this time frame. Similar results were obtained in a second experiment (not shown). There was also a statistically significant reduction in the mean number of histologically confirmed metastases per lung in the DNR group $(P=0.005$, nonparametric Mann-Whitney test) (Figure $5 \mathrm{~b})$. These data suggest that in progressing from lowto high-grade breast cancers, the tumor-suppressor effects of TGF- $\beta$ have been lost and prometastatic effects have been uncovered.

\section{Figure 5}

Decreased TGF- $\beta$ responsiveness does not affect primary tumorigenesis but suppresses metastasis in the high-grade breast carcinoma line M-IV. (a) Tumor growth kinetics in vivo. Nude mice were inoculated subcutaneously on each hind flank with retrovirally transduced M-IV cells $\left(2 \times 10^{5}\right.$ cells/site). For M-IV CON, $n=11$ sites injected; M-IV $\mathrm{DNR}, n=11$. Untransduced parental M-IV cells gave essential identical results to M-IV CON ( $n=4$, not shown). (b) Lung metastases. Metastatic efficiency was determined by quantitation of histologically detectable lung metastases 8 weeks after injection of $10^{6}$ retrovirally transduced cells into the tail vein of nude mice. Results are the mean \pm SD for $n=5$ (M-IV CON) and $n=10$ (M-IV DNR). CON, cells transduced with pLPCX; DNR, cells transduced with pLPC-DNR.

\section{Discussion}

The hypothesis that TGF- $\beta$ functions as a tumor suppressor early in the carcinogenic process but switches to a pro-oncogenic agent in late-stage disease has been widely proposed to reconcile much of the otherwise conflicting clinical and experimental data that exists in the literature (reviewed in refs. 2-4). Here we have demonstrated, we believe for the first time, that the biological output of the endogenous TGF- $\beta$-signaling network can indeed switch from tumor suppression to promotion of metastasis during the course of carcinogenic progression within a single cell lineage initiated by a single, defined oncogenic event (see Figure 6). In the model of breast cancer progression that we have used, this switch in biological readout occurs at the transition from histologically low-grade to high-grade breast cancer. The results therefore suggest that the final stages of carcinogenic progression may specifically involve genetic and/or epigenetic changes that radically alter the cellular interpretation of the TGF- $\beta$ signal.

Mechanistically, this switch from tumor suppressor to prometastatic factor does not appear to involve a qualitatively altered proliferative response, because

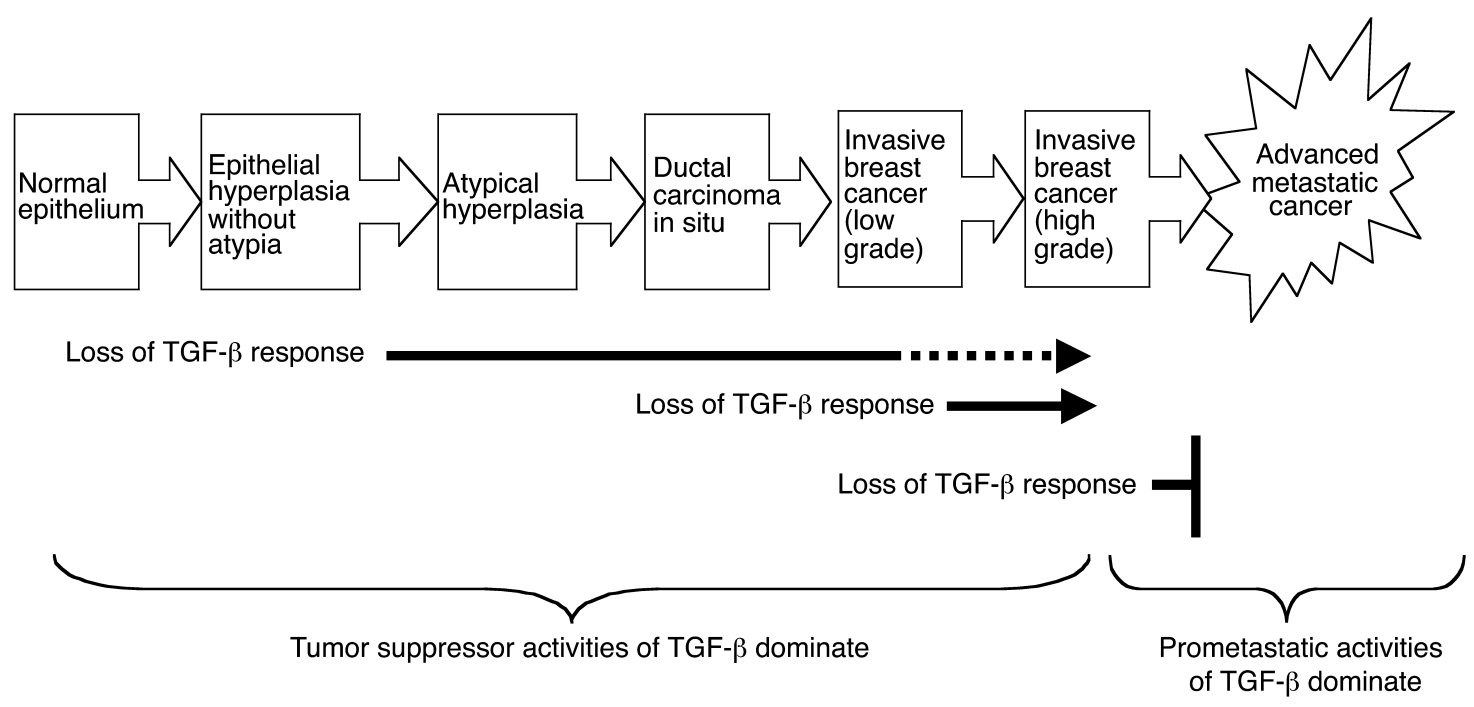

Figure 6

Summary of effect of decreased TGF- $\beta$ response at different stages in the carcinogenic process for the breast. 
TGF- $\beta$ was growth inhibitory in vitro for all four cell lines of the breast cancer progression series, regardless of whether TGF- $\beta$ exerted a tumor suppressor or prometastatic effect in vivo. In this respect, our model system differs from previous work by Schwarz et al., in which TGF- $\beta$ was shown to inhibit the in vitro proliferation of nonmetastatic fibroblasts, but to stimulate proliferation of their metastatic derivatives (18). Whether the different results reflect a fundamental difference in metastatic mechanisms between carcinomas and tumors of mesenchymal origin is not clear. We also did not see any major differences in vitro between the four cell lines in terms of their ability to activate the Smad signal transduction pathways or to regulate the expression of prototypical TGF- $\beta$-regulated genes such as fibronectin and c-myc. Thus, the in vitro biology of the cell lines is currently giving no insight into mechanisms underlying the "metastatic switch" in vivo. Tumors in vivo are complex "organs" composed of many cell types, and it is known that tumor stroma plays a very important role in modulating invasion and metastasis $(19,20)$. We are actively investigating the possibility that the low- and high-grade breast cancer cell lines interact differently with the host environment, such that a different biological readout to TGF- $\beta$ is obtained in the two cell types in vivo.

Several important points about the biology of TGF- $\beta$ in the different stages of breast cancer progression can be deduced from our data that shed light on existing clinical observations. Firstly, reduction of TGF- $\beta$ response in the immortalized but otherwise normal breast epithelial cell line MCF10A (M-I) did not give rise to tumors or even to benign proliferative lesions. This result suggests that reduced TGF- $\beta$ responsiveness is not an initiating lesion for breast cancer, even in the context of an immortalized cell. Consistent with this finding, decreased expression of T $\beta R I I$ is seen in some morphologically normal human breast epithelia (7). Our work suggests that this loss may be without immediate consequence until additional mutational or epigenetic perturbations occur.

Once an initiating oncogenic event does occur, however, our data clearly show that reduction of TGF- $\beta$ responsiveness in the premalignant breast epithelium can dramatically increase the risk of subsequent tumor formation. Alone, the presence of activated Ha-ras in the M-II premalignant cells causes these cells to form lesions with features of proliferative breast disease, and during the 2-year lifetime of the mouse, about $20-25 \%$ of these lesions will finally progress to invasive carcinoma (14). The combination of diminished TGF- $\beta$ response and activated HRAS, however, caused carcinomas to form at $40-60 \%$ of the sites injected within 65 days, during which time none of the cells with normal TGF- $\beta$ response formed tumors. These data are in agreement with our previous work showing that transgenic overexpression of the DNR in the mouse mammary gland, while not affecting spontaneous mammary tumorigenesis, did increase tumorigenesis following initiation with a chemical carcinogen (21). Similar results have been seen in the skin, liver, stomach, prostate, and intestine $(17,22-24)$. Clearly, it will be important to determine the spectrum of oncogenic lesions that can cooperate with loss of TGF- $\beta$ response to cause malignant conversion in the breast and other epithelia. Our data strongly suggest, however, that the reduced expression of T $\beta$ RII, seen clinically in a subset of human breast epithelial hyperplasias without atypia (7), may be a marker for increased risk because of the increased probability of malignant conversion in the presence of initiating oncogenic lesions.

Not only does diminished TGF- $\beta$ response accelerate malignant conversion in the early stages of breast carcinogenesis, but it also has an effect later in the process. Loss of TGF- $\beta$ response in the cell line that forms relatively well-differentiated carcinomas (M-III) resulted in an increased tumor growth rate, more rapid proliferation, and a more aggressive morphology. In a recent clinical study, decreased T $\beta$ RII expression in DCIS and in invasive breast cancer correlated with higher mitotic index and higher tumor grade (8). Our data strongly support a causal connection between the loss of TGF- $\beta$ response and a more aggressive tumor phenotype at these stages in breast carcinogenesis.

Interestingly, in the clinical study cited above, there was no significant correlation between decreased T $\beta$ RII expression in the primary breast tumor and lymph node status (8). This raises the possibility that although loss of TGF- $\beta$ response increases growth rate and causes a more aggressive morphology in the primary tumor, it does not promote metastatic spread. In support of this idea, we have shown that loss of TGF- $\beta$ response in the highly aggressive metastatic cell line (M-IV) had no effect on primary tumorigenesis, but actually decreased metastatic efficiency. We are currently probing the underlying mechanisms, but data in other systems suggest that TGF- $\beta$ effects on motility and invasiveness, and possibly survival of the tumor cell, may contribute $(25,26)$. Our observations are entirely consistent with the hypothesis that in latestage disease, TGF- $\beta$ switches from suppressor to promoter of carcinogenesis. Data in support of this hypothesis have also been gathered in the skin (27). That study, however, involved ectopic overexpression of TGF- $\beta 1$, and it was not clear whether endogenous TGF- $\beta$ in the skin would behave similarly. To the best of our knowledge, ours is the first demonstration of the switch in a system that allows the differing roles of endogenous TGF- $\beta$ at multiple stages of carcinogenic progression to be cleanly dissected out and the stage of the switch to prometastatic factor to be identified.

Our observations have potential implications for disease management in breast cancer. Decreased T $\beta R I I$ expression in early proliferative lesions of the breast is fairly common, and this expression pattern identifies a high-risk subgroup (7). Since we have shown that loss of TGF- $\beta$ response in premalignant breast cells increases the probability of malignant conversion, our data 
suggest that strategies to restore TGF- $\beta$ responsiveness in early proliferative lesions might be effective in preventing or delaying subsequent development of invasive breast cancer. In human breast cancer cell lines with decreased T $\beta$ RII, treatment with chromatin-modifying agents can restore T $\beta$ RII expression $(28,29)$. Less-toxic strategies for reversing this epigenetic reduction in T $\beta$ RII or, alternatively, for augmenting residual TGF- $\beta$ responsiveness are under development and might ultimately be useful for early prevention $(30,31)$. In contrast, restoration of TGF- $\beta$ responsiveness in late-stage disease would be inadvisable because of the risk of promoting metastasis. At this stage, however, antagonism of the TGF- $\beta$ ligand shows promise as a therapeutic strategy $(25,32)$.

In conclusion, we have used a xenograft model of breast cancer progression to show that loss of TGF- $\beta$ response is causally involved in promoting malignant transformation and disease progression up to the stage of high-grade invasive breast cancer. At this late stage, loss of TGF- $\beta$ response actually prevents further progression by suppressing metastatic spread. This model represents a valuable resource to mine for information about the mechanisms that underlie the opposing roles of TGF- $\beta$ s at different stages in breast cancer progression and the changes in molecular and biological context that cause TGF- $\beta$ to switch from tumor suppressor to prometastatic factor in late-stage disease.

\section{Acknowledgments}

We acknowledge the excellent technical support of Mario Anzano and Jeff Nyswaner for animal husbandry and of the staff of the Pathology/Histotechnology Laboratory. We thank Anita Roberts and Glenn Merlino for helpful discussions and Michael Reiss for anti-phospho-Smad3 Ab. This project has been funded in part with federal funds from the National Cancer Institute, NIH, under contract N01-C0-12400. The content of this publication does not necessarily reflect the views or policies of the Department of Health and Human Services, nor does mention of trade names, commercial products, or organizations imply endorsement by the U.S. government.

1. Roberts, A.B., and Sporn, M.B. 1990. The transforming growth factors- $\beta$. In Handbook of experimental pharmacology. Peptide growth factors and their receptors. M.B. Sporn and A.B. Roberts, editors. Springer-Verlag. Berlin, Germany. 419-472.

2. Derynck, R., Akhurst, R.J., and Balmain, A. 2001. TGF- $\beta$ signaling in tumor suppression and cancer progression. Nat. Genet. 29:117-129.

3. Wakefield, L.M., and Roberts, A.B. 2002. TGF- $\beta$ signaling: positive and negative effects on tumorigenesis. Curr. Opin. Genet. Dev. 12:22-29.

4. Massague, J., Blain, S.W., and Lo, R.S. 2000. TGF- $\beta$ signaling in growth control, cancer, and heritable disorders. Cell. 103:295-309.

5. Fynan, T.M., and Reiss, M. 1993. Resistance to inhibition of cell growth by transforming growth factor- $\beta$ and its role in oncogenesis. Crit. Rev. Oncog. 4:493-540.

6. Gold, L.I. 1999. The role for transforming growth factor- $\beta$ (TGF- $\beta$ ) in human cancer. Crit. Rev. Oncog. 10:303-360.

7. Gobbi, H., et al. 1999. Transforming growth factor- $\beta$ and breast cancer risk in women with mammary epithelial hyperplasia. J. Natl. Cancer Inst. 91:2096-2101.
8. Gobbi, H., et al. 2000. Loss of expression of transforming growth factor $\beta$ type II receptor correlates with high tumour grade in human breast insitu and invasive carcinomas. Histopathology. 36:168-177.

9. Dalal, B.I., Keown, P.A., and Greenberg, A.H. 1993. Immunocytochemical localization of secreted transforming growth factor- $\beta 1$ to the advancing edges of primary tumors and to lymph node metastases of human mammary carcinoma. Am. J. Pathol. 143:381-389.

10. Walker, R.A., and Dearing, S.J. 1992. Transforming growth factor $\beta 1$ in ductal carcinoma in situ and invasive carcinomas of the breast. Eur. J. Cancer. 28:641-644.

11. Gorsch, S.M., Memoli, V.A., Stukel, T.A., Gold, L.I., and Arrick, B.A. 1992. Immunohistochemical staining for transforming growth factor $\beta 1$ associates with disease progression in human breast cancer. Cancer Res. 52:6949-6952.

12. Kong, F.M., et al. 1995. Elevated plasma transforming growth factor- $\beta 1$ levels in breast cancer patients decrease after surgical removal of the tumor. Ann. Surg. 222:155-162.

13. Soule, H.D., et al. 1990. Isolation and characterization of a spontaneously immortalized human breast epithelial cell line, MCF-10. Cancer Res. 50:6075-6086.

14. Dawson, P.J., Wolman, S.R., Tait, L., Heppner, G.H., and Miller, F.R. 1996. MCF10AT: a model for the evolution of cancer from proliferative breast disease. Am. J. Pathol. 148:313-319.

15. Santner, S.J., et al. 2001. Malignant MCF10CA1 cell lines derived from premalignant human breast epithelial MCF10AT cells. Breast Cancer Res. Treat. 65:101-110.

16. Pear, W.S., Nolan, G.P., Scott, M.L., and Baltimore, D. 1993. Production of high-titer helper-free retroviruses by transient transfection. Proc. Natl. Acad. Sci. U. S. A. 90:8392-8396.

17. Tang, B., et al. 1999. Loss of responsiveness to transforming growth factor- $\beta$ induces malignant transformation of nontumorigenic rat prostate epithelial cells. Cancer Res. 59:4834-4842.

18. Schwarz, L.C., Gingras, M.C., Goldberg, G., Greenberg, A.H., and Wright, J.A. 1988. Loss of growth factor dependence and conversion of transforming growth factor- $\beta 1$ inhibition to stimulation in metastatic Haras-transformed murine fibroblasts. Cancer Res. 48:6999-7003.

19. Bissell, M.J., and Radisky, D. 2001. Putting tumours in context. Nat. Rev. Cancer. 1:46-54.

20. Liotta, L.A., and Kohn, E.C. 2001. The microenvironment of the tumourhost interface. Nature. 411:375-379.

21. Bottinger, E.P., Jakubczak, J.L., Haines, D.C., Bagnall, K., and Wakefield, L.M. 1997. Transgenic mice overexpressing a dominant-negative mutant type II transforming growth factor $\beta$ receptor show enhanced tumorigenesis in the mammary gland and lung in response to the carcinogen 7,12-dimethylbenz-[a]-anthracene. Cancer Res. 57:5564-5570.

22. Amendt, C., Schirmacher, P., Weber, H., and Blessing, M. 1998. Expression of a dominant negative type II TGF- $\beta$ receptor in mouse skin results in an increase in carcinoma incidence and an acceleration of carcinoma development. Oncogene. 17:25-34.

23. Kanzler, S., et al. 2001. Hepatocellular expression of a dominant-negative mutant TGF- $\beta$ type II receptor accelerates chemically induced hepatocarcinogenesis. Oncogene. 20:5015-5024.

24. Hahm, K.B., et al. 2002. Conditional loss of TGF- $\beta$ signalling leads to increased susceptibility to gastrointestinal carcinogenesis in mice. Aliment. Pharmacol. Ther. 2(Suppl. 16):115-127.

25. Muraoka, R.S., et al. 2002. Blockade of TGF- $\beta$ inhibits mammary tumor cell viability, migration, and metastases. J. Clin. Invest. 109:1551-1559. doi:10.1172/JCI200215234.

26. Oft, M., Heider, K.H., and Beug, H. 1998. TGF- $\beta$ signaling is necessary for carcinoma cell invasiveness and metastasis. Curr. Biol. 8:1243-1252.

27. Cui, W., et al. 1996. TGF $\beta 1$ inhibits the formation of benign skin tumors, but enhances progression to invasive spindle carcinomas in transgenic mice. Cell. 86:531-542.

28. Lee, B.I., et al. 2001. MS-275, a histone deacetylase inhibitor, selectively induces transforming growth factor $\beta$ type II receptor expression in human breast cancer cells. Cancer Res. 61:931-934.

29. Liu, Y., Zhong, X., Li, W., Brattain, M.G., and Banerji, S.S. 2000. The role of $\mathrm{Sp} 1$ in the differential expression of transforming growth factor- $\beta$ receptor type II in human breast adenocarcinoma MCF-7 cells. J. Biol. Chem. 275:12231-12236.

30. Law, B.K., et al. 2002. Rapamycin potentiates transforming growth factor $\beta$-induced growth arrest in nontransformed, oncogene-transformed, and human cancer cells. Mol. Cell Biol. 22:8184-8198.

31. Suh, N., et al. 2003. Synthetic triterpenoids enhance transforming growth factor $\beta /$ Smad signaling. Cancer Res. 63:1371-1376.

32. Yang, Y.A., et al. 2002. Lifetime exposure to a soluble TGF- $\beta$ antagonist protects mice against metastasis without adverse side effects. J. Clin. Invest. 109:1607-1615. doi:10.1172/JCI200215333. 